\title{
Fully Compressible Low-Mach Number Simulations of Carbon-dioxide at Supercritical Pressures and Trans-critical Temperatures
}

\author{
Uttiya Sengupta $^{1}$ (D) Hassan Nemati ${ }^{1}$. \\ Bendiks J. Boersma ${ }^{1} \cdot$ Rene Pecnik $^{1}$
}

Received: 17 March 2017 / Accepted: 30 October 2017 / Published online: 21 November 2017

(C) The Author(s) 2017. This article is an open access publication

\begin{abstract}
This work investigates fully developed turbulent flows of carbon-dioxide close to its vapour-liquid critical point in a channel with a hot and a cold wall. Two direct numerical simulations are performed at low Mach numbers, with the trans-critical transition near the channel centre and the cold wall, respectively. An additional simulation with constant transport properties is used to selectively investigate the effect of the non-linear equation of state on turbulence. Compared to the case where the pseudo-critical transition occurs in the channel center, the case with the pseudo-critical transition close to the cold wall reveals that compressibility effects can exist in the near-wall region even at low Mach numbers. An analysis of the velocity streaks near the hot and the cold walls also indicates a greater degree of streak coherence near the cold wall. A comparison between the constant and variable viscosity cases at the same Reynolds number, Mach number and having the same isothermal wall boundary conditions reveals that variable viscosity increases turbulence near the cold wall and also causes higher velocity gradients near the hot wall. We also show that the extended van Driest transformation results in a better agreement of the velocity profile with the log-law of the wall compared to the standard van Driest transformation. The semilocally scaled turbulent velocity fluctuations and the turbulent kinetic energy budgets on the hot and the cold sides of the channel collapse on top of each other, thereby establishing the validity of Morkovin's hypothesis.
\end{abstract}

Uttiya Sengupta

sumeetsengupta@gmail.com

Rene Pecnik

r.pecnik@tudelft.nl

Hassan Nemati

h.nemati@tudelft.nl

Bendiks J. Boersma

b.j.boersma@tudelft.nl

1 Department of Process and Energy, Delft University of Technology, Leeghwaterstraat 39,

2628 CB, Delft, Netherlands 
Keywords Direct numerical simulation - Supercritical fluid · Trans-critical transition . Peng Robinson equation · Solenoidal dissipation · Extended van Driest transformation

\section{Introduction}

Fluids at temperatures and pressures above their vapour-liquid critical point are known as supercritical fluids. Above the vapour-liquid critical point, the heat capacity of a supercritical fluid at constant pressure $\left(C_{p}\right)$ shows a maximum value at a certain temperature, known as the pseudo-critical temperature $\left(T_{p c}\right)$. This point is called the pseudo-critical point. The line joining the pseudo-critical points for a range of pressures above the critical point is called the pseudo-critical line. Below the critical point, the fluid exists as a mixture of liquid and vapour in the two phase region. The line separating the supercritical fluid from the two phase region consists of the saturation liquid and the saturation vapour lines. Very near to the critical point, the fluid can neither be called a liquid nor a gas and has a unique ombination of gas-like and liquid-like properties. The area bounded by the critical isobar and the critical isotherm represents supercritical fluids, as shown in Fig. 1. The pressure and specific volume are normalized by the critical pressure $\left(p_{c}\right)$ and the critical volume $\left(v_{c}\right)$ of $\mathrm{CO}_{2}$, respectively. Along any isobar above the critical point, on crossing the pseudo-critical point, all the thermodynamic and transport properties like density, isobaric heat capacity, viscosity and thermal conductivity etc., exhibit sharp gradients for small changes in temperature. This phenomena is called trans-critical transition. This has been shown in Fig. 2 by

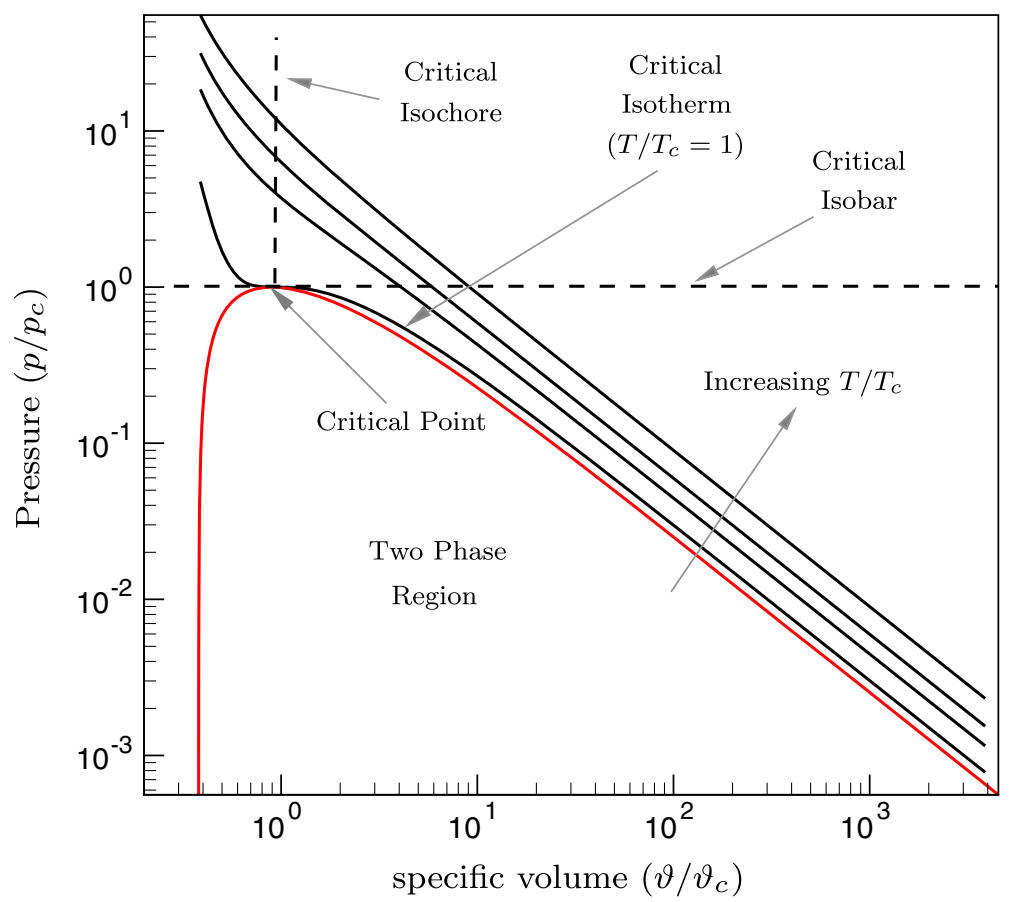

Fig. 1 Pressure as a function of specific volume normalized by their critical values at constant temperatures for $\mathrm{CO}_{2} ;(\longrightarrow) T / T_{c}=1.0 ; T / T_{c}=1.5, T / T_{c}=2.0, T / T_{c}=3.0 ;(\longrightarrow)$ saturation lines 
Fig. 2 Thermodynamic and transport properties of $\mathrm{CO}_{2}$ normalized by their values at the reference conditions as a function of temperature at a pressure of 80 bar. (- - heat capacity $\left(C_{p} / C_{p 0}\right) ;(-)$ density $\left(\rho / \rho_{0}\right) ;(\longrightarrow)$ viscosity $\left(\mu / \mu_{0}\right) ;(---)$ thermal conductivity $\left(\kappa / \kappa_{0}\right)$

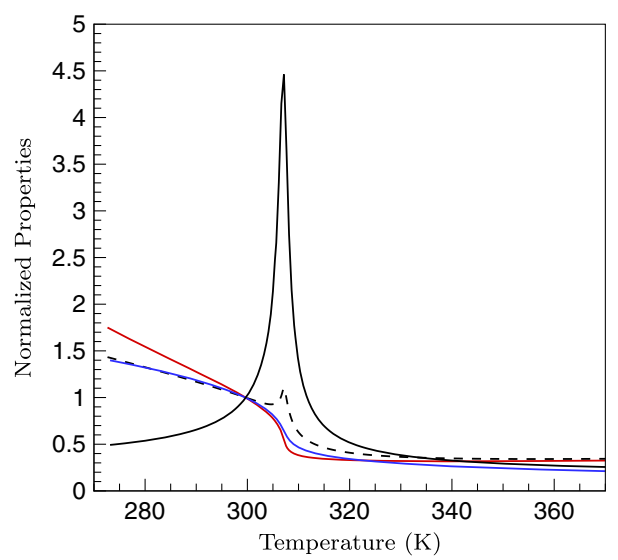

plotting the density $(\rho)$, heat capacity $\left(C_{p}\right)$, viscosity $(\mu)$ and the thermal conductivity $(\kappa)$ for supercritical $\mathrm{CO}_{2}$ at 80 bar as a function of temperature, normalized by their values at the reference condition of $80 \mathrm{bar}$ and $300 \mathrm{~K}$. The reference values for these properties are mentioned in Table 1 . The density and the heat capacity are calculated from the Peng Robinson equation of state and the viscosity and the thermal conductivity are calculated using the Refprop library [1]. Due to these unique characteristics, supercritical fluids have been the subject of research for a considerable period of time. These fluids have various applications in the industry, such as in supercritical power cycles, in drug delivery systems, as solvents in the extraction of plant materials and also as solvents in the extraction of polymers.

In many of the engineering applications of supercritical fluids mentioned above, buoyancy can play a role. Our simulations, however, have not taken into account the effects of buoyancy. A discussion is henceforth provided to include some of the effects of buoyancy in turbulent flows. Publications by previous authors, such as, [2, 3] have discussed in detail the changes in the nature of turbulence in channel flows and also in Poiseuille-RayleighBernard (PRB) flows due to buoyancy. This involved performing DNS under the assumption of temperature independent fluid properties, i.e. under the Oberbeck-Boussinesq hypothesis $(\mathrm{OB})$ and comparing the results to simulations with viscosity $(\mu)$ and thermal expansion coefficient $(\beta)$ varying with temperature. In case of PRB flows, the wall-normal transport of momentum and heat is increased by buoyancy compared to the case of forced convection where temperature is a passive scalar and the mixing efficiency increases with the increase in Richardson number $\left(R i_{\tau}\right)$. In case of a stable stratified flow in a channel with a top hot wall, a bottom cold wall and gravity acting downward in the wall-normal direction, it is seen that the fluid particles near the hot wall experience a larger buoyancy force resulting in a partial laminarization of the flow near the hot wall. If the location of the hot and cold walls are reversed, then it would result in unstable stratification. This is in contrast to forced

Table 1 Density $\left(\rho_{0}\right)$, Heat capacity $\left(C_{p 0}\right)$, viscosity $\left(\mu_{0}\right)$ and thermal conductivity $\left(\kappa_{0}\right)$ for supercritical $\mathrm{CO}_{2}$ under the reference conditions of 80 bar and $300 \mathrm{~K}$

\begin{tabular}{llll}
\hline$\rho_{0}$ & $C_{p 0}$ & $\mu_{0}$ & $\kappa_{0}$ \\
\hline $689.46 \mathrm{~kg} / \mathrm{m}^{3}$ & $4.98 \mathrm{~kJ} / \mathrm{kgK}$ & $6.093 * 10^{-5} \mathrm{~Pa} . \mathrm{s}$ & $8.246 * 10^{-2} \mathrm{~W} / \mathrm{mK}$ \\
\hline
\end{tabular}


convection flows, where only the wall temperatures are significant and the location of the hot and cold wall on the bottom or the top are irrelevant.

In case of turbulent flows of supercritical fluids, it is found that the sharp property variations alter the conventional behaviour of gas dynamics and turbulence. Direct numerical simulations for upward annular flows of supercritical $\mathrm{CO}_{2}$ with constant heat-flux wall boundary conditions was conducted by [4]. It was found that the most singular phenomenon is observed at supercritical pressures when there is trans-critical transition in the flow. This leads to some peculiar characteristics in the turbulent flow field, such as heat transfer deterioration causing the weakening of the velocity streaks near the point of the maxima of temperature fluctuation and also, the weakening of ejection and sweep motions near the wall. Large eddy simulation (LES) of a turbulent jet of nitrogen under supercritical pressure was performed by [5]. It compares low pressure LES simulations and real gas thermodynamics with data extracted from experiments. This approach has indicated that the high density gradients and non-linear thermodynamics has a limited impact on the jet spreading rate and the pseudo-similarity behaviour for jets. Turbulence models are still not capable of providing satisfactory results for wall-bounded turbulent flows with strong heat transfer, as shown in [6]. A review of the turbulent flows of supercritical fluids was conducted by [7] in order to study various effects like buoyancy, flow acceleration and heat transfer deterioration in supercritical fluid flows. A numerical method has however been provided by [8] for conducting high fidelity simulations for supercritical fluids involving trans-critical transition. [9] has investigated developing turbulent flows of supercritical $\mathrm{CO}_{2}$ in a pipe geometry involving forced and mixed convection. The effects of flow stratification caused by buoyancy for turbulent flows of supercritical $\mathrm{CO}_{2}$ in a pipe have been investigated by [10]. Large eddy simulations of cryogenic nitrogen injection have been performed by [11] using the Peng Robinson equation of state at supercritical pressures. In this paper, various subgrid-scale (SGS) models have been compared and the influence of the density gradients on the growth of instability have been investigated. One dimensional rarefraction properties in compression shocks have been investigated recently by [12] for non-ideal gases close to the vapour-liquid critical point. [13] also investigates the heat transfer characteristics in fully developed turbulent flows of $\mathrm{CO}_{2}$ in an annular geometry by simulating the low-Mach number approximation Navier-Stokes equations. In many previous works, such as [4, 7, 9] and [13], Navier-Stokes equations have been solved using the low-Mach number approximation, which takes into account changes in density due to changes in temperature, but ignores the effect of density changes due to the pressure variations. In order to further improve our understanding of the fluid dynamics of supercritical fluids and also to capture the compressibility effects, a fully compressible Navier-Stokes solver has been developed, which can include the effects of density changes due to the variation of both pressure and temperature. Cases have been simulated with isothermal wall boundary conditions such that we get trans-critical transition inside the flow field. Simulations with constant and variable transport properties have been compared in order to estimate the effects of variable viscosity. The effects of the changing location of trans-critical transition have been investigated by simulating a flow with $T_{p c}$ close to the cold wall.

This paper mainly analyzes the influence of trans-critical transition on near-wall turbulence and compressibility effects in fully developed turbulent flows of supercritical $\mathrm{CO}_{2}$ near the critical point at low Mach numbers. It further investigates the validity of scaling methodologies, such as the extended van Driest velocity scaling and the semi-local scaling for these flows. It is organized as follows: Section 2 shows the non-dimensionalized form of the compressible Navier-Stokes equations; Section 3 gives the details of various thermodynamic equations of state including the rationale for choosing Peng Robinson as the 
preferred equation of state, and the transport properties; Section 4 gives the numerical methods and simulation details; Section 5 gives the results and discussions; Section 6 gives the conclusions.

\section{Navier-Stokes Equations}

An in-house code has been developed to solve the compressible Navier-Stokes equations in Cartesian coordinates for fully developed turbulent flow in a channel. The continuity, momentum and energy equations are solved for the density $(\rho)$, the components of momentum in three directions $\left(\rho u_{i}\right)$ and the total energy $\left(\rho e_{0}\right)$, respectively. The total energy is expressed as $\rho e_{0}=\rho e_{i n t}+1 / 2 \rho u_{i}^{2}$, where $e_{i n t}$ is the internal energy and $1 / 2 \rho u_{i}^{2}$ is the kinetic energy. The non-dimensional Navier-Stokes equations are given as follows,

$$
\begin{gathered}
\frac{\partial \rho}{\partial t}+\frac{\partial \rho u_{j}}{\partial x_{j}}=0, \\
\frac{\partial \rho u_{i}}{\partial t}+\frac{\partial\left(\rho u_{i} u_{j}+p \delta_{i j}-\Gamma_{i j}\right)}{\partial x_{j}}=\rho f_{i}, \\
\frac{\partial \rho e_{0}}{\partial t}+\frac{\partial\left(u_{j} \rho e_{0}+u_{j} p+q_{j}-u_{i} \Gamma_{i j}\right)}{\partial x_{j}}=\rho u_{j} f_{j},
\end{gathered}
$$

with the viscous stress $\Gamma_{i j}$ and $q_{j}$ the heat flux defined as,

$$
\begin{gathered}
\Gamma_{i j}=\frac{\mu}{\operatorname{Re}}\left[\left(\frac{\partial u_{i}}{\partial x_{j}}+\frac{\partial u_{j}}{\partial x_{i}}\right)-\frac{2}{3} \delta_{i j} \frac{\partial u_{k}}{\partial x_{k}}\right], \\
q_{j}=-\frac{\kappa}{\operatorname{RePr} M_{p s}^{2}} \frac{\partial T}{\partial x_{j}} .
\end{gathered}
$$

In the above, $\mu$ is the viscosity, $p$ denotes the pressure, $\kappa$ is the thermal conductivity and $f_{i}$ is the applied forcing term in streamwise direction for the conservation of mass flux. The applied forcing term is analogous to $(-1 / \rho) \partial p / \partial x$ for pressure driven flows and is calculated in a way such that the quantities $\int \rho d y$ and $\int \rho U d y$ are conserved. The symbol $U$ refers to the velocity in the streamwise direction. The constant integrated density follows directly as a result of the continuity equation and the applied pressure gradient results in the conservation of the streamwise momentum. The non-dimensionalization is performed as follows.

$$
\begin{gathered}
x_{i}=\frac{x_{i}^{\star}}{H^{\star}}, \quad t=\frac{t^{\star} U_{b}^{\star}}{H^{\star}}, \quad e_{0}=\frac{e_{0}^{\star}}{U_{b}^{\star 2}}, \quad u_{i}=\frac{u_{i}^{\star}}{U_{b}^{\star}}, \\
p=\frac{p^{\star}}{\rho_{0}^{\star} U_{b}^{\star 2}}, \quad \rho=\frac{\rho^{\star}}{\rho_{0}^{\star}}, \quad \kappa=\frac{\kappa^{\star}}{\kappa_{0}^{\star}}, \quad T=\frac{T^{\star}}{T_{0}^{\star}}, \quad \mu=\frac{\mu^{\star}}{\mu_{0}^{\star}} .
\end{gathered}
$$

The superscript $\star$ is used to denote dimensional quantities. The non-dimensional numbers, namely, the Reynolds number, Prandtl number and Mach number are given as,

$$
R e=\frac{\rho_{0}^{\star} U_{b}^{\star} h^{\star}}{\mu_{0}^{\star}}, \quad \operatorname{Pr}=\frac{\mu_{0}^{\star} C_{p 0}^{\star}}{\kappa_{0}^{\star}}, \quad M=\frac{U_{b}^{\star}}{C_{0}^{\star}},
$$

where $C_{0}^{\star}$ is the speed of sound under the reference conditions. The pseudo-Mach number used to non-dimensionalize the heat conduction term is given as

$$
M_{p s}^{2}=\frac{U_{b}^{\star 2}}{C_{p 0}^{\star} T_{0}^{\star}} \text {. }
$$


The subscript 0 is used to denote the properties of $\mathrm{CO}_{2}$ under the reference conditions of 80 bar and $300 \mathrm{~K}$.

\section{Thermodynamic Equations of State and Transport Properties}

\subsection{Equation of state}

The choice of the equation of state is important as it is essential to accurately represent the thermodynamic properties of $\mathrm{CO}_{2}$ near the critical point in a computationally efficient manner. The equation of state can either be implemented directly in the Navier-Stokes solver or a lookup table approach can be used to tabulate the required thermodynamic properties that can then be obtained using an appropriate interpolation method. The thermodynamic properties, such as, pressure and temperature have to be evaluated in terms of the conservative variables density and internal energy. When the equation of state is implemented directly in the Navier-Stokes solver, temperature and pressure are evaluated by solving the departure function for internal energy by using a Newton-Raphson solver. Multiparameter equations of state, such as, [14] and [15] have a high level of accuracy near the critical point of $\mathrm{CO}_{2}$. However, these equations of state involve complicated terms and implementing the Newton-Raphson solver can be computationally very expensive. The lookup table approach suffers from the problem of consistency as normal interpolation methods are not consistent upto the $2^{\text {nd }}$ order derivative for these properties, as mentioned by [16]. In order to reach a compromise between accuracy, computational efficiency and consistency, a cubic equation of state is used, which is not as accurate as the multiparameter equations but is computationally more efficient. It also does not suffer from the problem of consistency like the lookup table approach. The Peng Robinson equation of state has been selected after careful consideration.

The cubic equation of state (EOS) to be used is chosen by comparing the accuracy of the thermodynamic properties predicted by the different cubic equations near the critical point of $\mathrm{CO}_{2}$. The thermodynamic properties like density and heat capacity $\left(C_{p}\right)$ predicted by the van der Waals (VdW), Peng Robinson (PR) and Redlich Kwong (RK) equation of state at constant pressure of 80 bar are compared with the actual values extracted from the Refprop library.

In order to calculate thermodynamic properties from a real gas, the departure function for that property with respect to the chosen equation of state has to be evaluated. The departure function for any thermodynamic property of a real gas is defined as the difference in the value of that property determined from the chosen real gas equation of state and the value of the same property for an ideal gas under the same conditions of temperature and pressure. Mathematically, it can be expressed as follows,

$$
X^{R}(T, P)=X(T, P)-X_{i g}(T, P),
$$

where $X(T, P)$ is a thermodynamic property of the real gas at temperature $(T)$ and pressure $(P), X_{i g}(T, P)$ is the same property for the ideal gas (IG) and $X^{R}(T, P)$ is the departure function under the same conditions of temperature and pressure. For the compressible Navier-Stokes solver, the energy equation calculates the total energy. The kinetic energy is then subtracted from the total energy to determine the internal energy. The calculation of departure functions for the internal energy from the van der Waals and the Redlich Kwong equation of state is mentioned in Appendix A. The departure function for the Peng Robinson equation of state is derived as follows. 
The non-dimensional Peng Robinson equation of state is given as

$$
P=\frac{R T}{\vartheta-b}-\frac{a \alpha}{\vartheta^{2}+2 b \vartheta-b^{2}},
$$

where the non-dimensionalization is performed by

$$
R=\frac{R^{\star} T_{0}^{\star}}{U_{0}^{\star 2}} ; a=\frac{a^{\star} \rho_{0}^{\star}}{U_{0}^{\star 2}} ; b=b^{\star} \rho_{0}^{\star},
$$

with $\vartheta=1 / \rho, a^{\star}=0.457235 R^{\star 2} T_{c}^{\star 2} / P_{c}^{\star}$ and $b^{\star}=0.077796 R^{\star} T_{c}^{\star} / P_{c}^{\star}$. The parameter $\alpha$ is determined by the acentric factor $\omega$ and the reduced temperature $T_{r}=T / T_{c}$ as,

$$
\alpha=\left(1+K\left(1-T_{r}^{0.5}\right)\right)^{2}, K=0.37464+1.54226 \omega-0.26992 \omega^{2} .
$$

The residual internal energy, $U^{R}$, is given as:

$$
U^{R}=U-U^{i g}=\int_{\infty}^{\vartheta}\left(T\left(\frac{\partial S}{\partial \vartheta}\right)_{T}-P\right) d \vartheta
$$

Substituting Maxwell's relations, $(\partial S / \partial \vartheta)_{T}=(\partial P / \partial T)_{\vartheta}$, in Eq. 13 we obtain

$$
\begin{gathered}
U^{R}=\int_{\infty}^{\vartheta}\left[T\left(\frac{\partial P}{\partial T}\right)_{\vartheta}-P\right] d \vartheta, \\
U-C_{\vartheta} T=\int_{\infty}^{\vartheta} \frac{a\left(\alpha-T \frac{d \alpha}{d T}\right)}{2 \sqrt{2} b}\left[\frac{1}{(\vartheta+b-\sqrt{2} b)}-\frac{1}{(\vartheta+b+\sqrt{2} b)}\right] \\
U=C_{\vartheta} T+\frac{a\left(\alpha-T \frac{d \alpha}{d T}\right)}{2 \sqrt{2} b} \log \frac{(1+b(1-\sqrt{2}) \rho)}{(1+b(1+\sqrt{2}) \rho)} .
\end{gathered}
$$

The enthalpy is related to internal energy as $H^{0}=U+P / \rho$ and the heat capacity at constant pressure is $C_{p}=\left(\partial H^{0} / \partial T\right)_{p}$. The plots in Fig. 3a and b showing the density and the heat capacity at constant pressure $\left(C_{p}\right)$ for supercritical $\mathrm{CO}_{2}$ at 80 bar as a function of temperature, establish that the Peng Robinson equation of state represents the actual properties obtained from the Refprop library far more accurately than the other cubic equations of state, such as van der Waals equation and the Redlich Kwong equation of state. Hence, the Peng Robinson equation is chosen to calculate the thermodynamic properties in the Navier-Stokes solver.

\subsection{Implementation of the Peng Robinson equation}

The non-dimensionalized departure function for the Peng Robinson equation of state is given as

$$
U=\frac{R T_{r} T_{c}}{\gamma-1}+\frac{(1+K)\left(1+K\left(1-T_{r}^{0.5}\right)\right) a}{2 \sqrt{2} b} \log \left(\frac{1+b(1-\sqrt{2}) \rho}{1+b(1+\sqrt{2}) \rho}\right)
$$



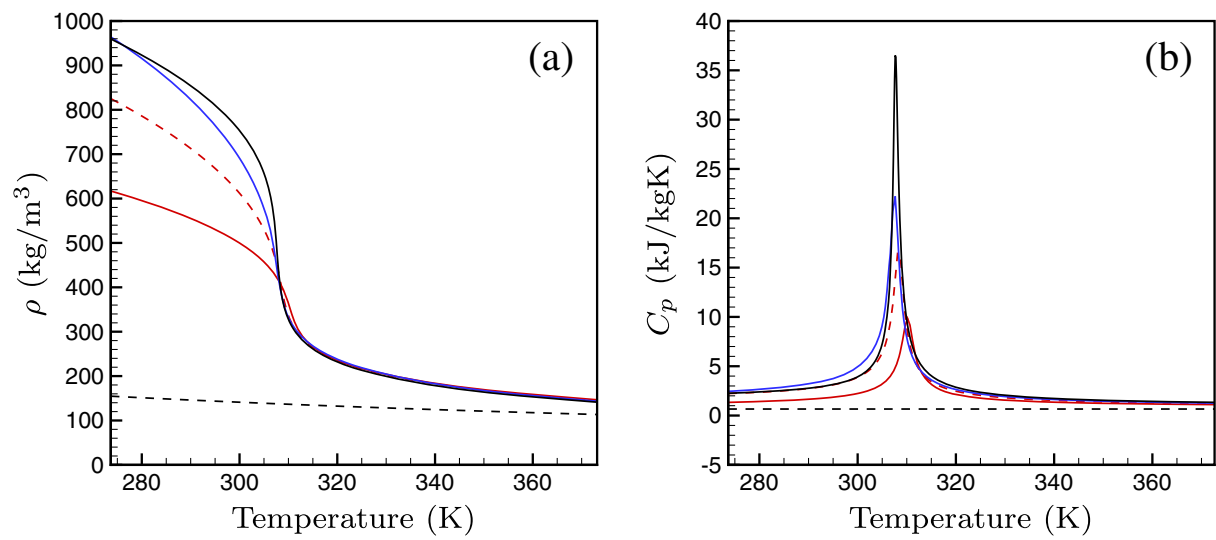

Fig. 3 Density as a function of temperature for supercritical $\mathrm{CO}_{2}$ at $80 \mathrm{bar}$ for different cubic equations of state compared to that from the Refprop library [1]. (- - - ) IG, ( -$) \mathrm{VdW},(---) \mathrm{RK},(-\square)$ PR, ( - $)$ Refprop

where $\gamma=R / C_{\vartheta}+1$, and $T_{r}=T / T_{c}$ is the reduced non-dimensional temperature. It is evident that the equation above is quadratic in $\sqrt{T_{r}}$. It can be represented as

$$
A{\sqrt{T_{r}}}^{2}+B \sqrt{T_{r}}+C=0,
$$

where $A, B$ and $C$ are

$$
\begin{gathered}
A=\frac{R T_{c}}{\gamma-1}, \\
B=-\frac{K(1+K) a}{2 \sqrt{2} b} \log \left(\frac{1+b(1-\sqrt{2}) \rho}{1+b(1+\sqrt{2}) \rho}\right), \\
C=\frac{(1+K)^{2} a}{2 \sqrt{2} b} \log \left(\frac{1+b(1-\sqrt{2}) \rho}{1+b(1+\sqrt{2}) \rho}\right)-U .
\end{gathered}
$$

The analytical formula for finding the roots of a quadratic equation is used to determine the roots. Two positive roots are not possible for this equation as it is thermodynamically not possible to have the same internal energy for a gas at a particular density and two different temperatures. Therefore, one of the roots is positive and the other is negative. The negative root is frivolous and is neglected. We can thus arrive at a unique solution for the temperature. This methodology is adopted to avoid iterations with a Newton-Raphson solver and to arrive at the solution in a computationally efficient manner.

\subsection{Transport properties}

The Refprop library calculates transport properties, such as viscosity and thermal conductivity using the transport models developed by $[17,18]$. Instead of directly calling Refprop, the transport properties are tabulated within a relevant range for temperature and pressure and are then stored in a lookup table. For any point inside the flow domain, transport properties like viscosity and thermal conductivity are uniquely determined at each point by calculating the indices in the lookup table and using a $2^{\text {nd }}$ order bilinear interpolation method to interpolate between the closest points. The lookup table approach for calculating the transport 
properties is not computationally expensive, as it involves a cartesian table in temperature and pressure, which are previously calculated using the Peng Robinson equation of state. As temperature and pressure are both known inputs to the table, the indices can be calculated directly without employing a search algorithm.

\section{Numerical Methods and Simulated Cases}

The turbulent channel flow geometry is given in Fig. 4. The channel is periodic in streamwise and spanwise directions with streamwise length $L_{x}=4 \pi H$, spanwise length $L_{z}=$ $2 \pi H$ and wall normal height $L_{y}=2 H$, where $H$ is the half channel height. The number of grid points used is $720 \times 720 \times 360$ in the streamwise, spanwise and wall-normal directions, respectively. In streamwise and spanwise directions, the mesh is uniform and pseudo spectral methods are used to calculate the derivatives using a Fast Fourier Transform (FFT) library. In order to minimize aliasing errors, the derivatives for the advective terms in the periodic directions are calculated using the quasi skew-symmetric method developed by [19]. In the wall-normal direction, the mesh is fully collocated and a finite volume scheme is used in which the derivatives for the advective and the diffusive terms are obtained by interpolating the quantities to the cell faces and then differentiating to obtain the values of the derivatives at the cell center. The interpolation and differentiation near the center of the channel are performed in accordance with the $6^{\text {th }}$ order compact finite difference method developed by [20] for collocated meshes. $4^{\text {th }}$ order boundary schemes also developed by [20] are used for implementing the Dirichlet boundary condition for temperature and velocity at the wall. In the course of our simulations, it is not necessary to calculate the density and pressure at the wall. In the energy equation, the pressure is multiplied with the velocity and the product vanishes at the walls due to the no slip boundary condition. However, the wall-normal gradient of pressure at the walls is required in the wall-normal momentum equation, which is set to zero as an approximation. The terms involving density in the continuity, momentum and energy equations $\left(\rho u_{i}, \rho u_{i} u_{j}, \rho u_{j} e_{0}\right)$ are always multiplied with velocities and therefore, the value of these terms at the walls is also equal to zero. For these terms, the $4^{\text {th }}$ order scheme for Dirichlet boundary conditions, developed by [20] can be used without calculating the pressure and density at the walls. The time integration is performed using an explicit third order Runge Kutta method as given by [21]. A hyperbolic tangent function is used to obtain a non-uniform mesh in the wall-normal direction. The domain is parallelized using 2DECOMP\&FFT library with 2D pencil decomposition. The

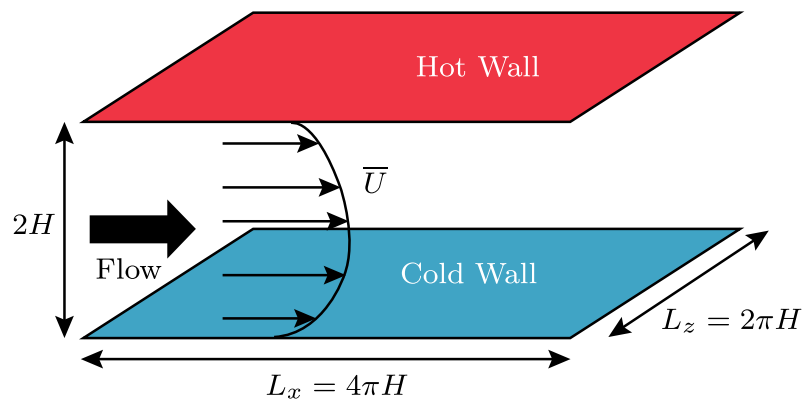

Fig. 4 Flow geometry for fully developed turbulent channel flow with wall temperature difference 
Table 2 Details of the simulations performed using supercritical $\mathrm{CO}_{2}$, stating cold and hot wall temperatures, $T_{w c}, T_{w h}$, viscosity and thermal conductivity, $\mu, \kappa$, and resulting friction Reynolds number at both walls

\begin{tabular}{lllll}
\hline Case & $T_{w c}, T_{w h}$ & Transport properties $\mu, \kappa$ & $R e_{\tau w c}$ & $R e_{\tau w h}$ \\
\hline $\mathrm{SC}_{1}$ & $300 \mathrm{~K}, 315 \mathrm{~K}$ & Tabulated Refprop data & 218.18 & 310.12 \\
$\mathrm{SC}_{2}$ & $306 \mathrm{~K}, 321 \mathrm{~K}$ & Tabulated Refprop data & 343.63 & 438.52 \\
$\mathrm{SCCTP}$ & $300 \mathrm{~K}, 315 \mathrm{~K}$ & Constant & 126.41 & 224.46 \\
\hline
\end{tabular}

code has been validated for fully developed turbulent flows of an ideal gas with the data published by [22] and [23]. This is shown in Appendix B.

Three cases are simulated which have a bulk Reynolds Number of 2800 (based on the reference conditions, bulk velocity and half channel height), bulk pressure $\left(P_{0}\right)$ of 80 bar, reference temperature $\left(T_{0}\right)$ of $300 \mathrm{~K}$, and a reference Mach number of 0.2. All simulations use the Peng Robinson equation of state to calculate the temperature and pressure. The Prandtl number at reference conditions is 3.019 and the speed of sound is $261.4 \mathrm{~m} / \mathrm{s}$. All the simulations are forced convection flows without the influence of gravity.

The differences between the three cases are highlighted in Table 2. The first two cases have variable transport properties tabulated from Refprop and are called supercritical variable property cases $\mathrm{SC}_{1}$ and $\mathrm{SC}_{2}$, respectively. The third case has constant viscosity and thermal conductivity calculated at the reference conditions and is called the supercritical constant transport property (SCCTP) case. The cases $\mathrm{SC}_{1}$ and SCCTP have isothermal wall boundary conditions with temperatures $300 \mathrm{~K}$ and $315 \mathrm{~K}$ at the two walls. For the case $\mathrm{SC}_{2}$, the wall temperatures are $306 \mathrm{~K}$ and $321 \mathrm{~K}$. At a pressure of 80 bar for carbon dioxide, the pseudo-critical temperature of $\mathrm{CO}_{2}$ is around $307.5 \mathrm{~K}$. Thus, the cases $\mathrm{SC}_{1}$ and SCCTP have the trans-critical transition roughly between the middle of the channel and the hot wall, whereas the case $\mathrm{SC}_{2}$ has trans-critical transition very close to the cold wall.

Table 3 reports mesh resolutions for all cases in terms of Kolmogorov and Batchelor scales. The Kolmogorov length scales, which indicate the resolution of the momentum scales are defined as $\eta=\sqrt[4]{\left((\bar{\mu} / \bar{\rho})^{3} \bar{\rho} / \epsilon\right)}$. Here, $\mu, \rho$ and $\epsilon$ refer to the viscosity, density and the dissipation of turbulent kinetic energy obtained from the turbulent kinetic energy budgets of the simulations, respectively. As the Prandtl number in supercritical fluids is more than unity, the mesh resolution in terms of Batchelor scales is also important as the Batchelor scales indicate the resolution of the thermal scales. The Batchelor scales are defined as: $\eta_{B}=\eta / \sqrt{P r}$, where Pr refers to the Prandtl number. The maximum mesh resolution in terms of the Kolmogorov length scales are within the limits $\Delta y<2 \eta, \Delta z<6 \eta$ and $\Delta x<12 \eta$, as specified by previous authors [24, 25].

Table 3 Spatial resolution with respect to Kolmogorov scales $(\eta)$ and Batchelor scales $\left(\eta_{B}\right)$ in streamwise, wall-normal and spanwise directions

\begin{tabular}{lllllll}
\hline Case & $\Delta x / \eta$ & $\Delta y_{\min } / \eta$ & $\Delta z / \eta$ & $\Delta x / \eta_{B}$ & $\Delta y_{\min } / \eta_{B}$ & $\Delta z / \eta_{B}$ \\
\hline $\mathrm{SC}_{1}$ & 3.60 & 0.62 & 1.80 & 5.30 & 0.96 & 2.65 \\
$\mathrm{SC}_{2}$ & 5.00 & 0.81 & 2.50 & 14.40 & 2.52 & 7.20 \\
$\mathrm{SCCTP}$ & 2.50 & 0.12 & 1.25 & 4.40 & 0.25 & 2.20 \\
\hline
\end{tabular}

Only maximum values are stated 
DNS data is extracted after a fully converged turbulent velocity and pressure field develops and time averaging is done using data for 10 flow through times at an interval of 2000 time steps for each of the cases mentioned above.

\section{Results and Discussion}

\subsection{Mean flow and turbulence statistics}

Averaged quantities for velocity, temperature, density, density rms, temperature rms and pressure rms are shown in Fig. 5 for all three cases. The mean velocity profile for case SCCTP is almost symmetric, while this is not the case for cases $\mathrm{SC}_{1}$ and $\mathrm{SC}_{2}$, shown in Fig. 5a. This is due to the decrease in viscosity with increase in temperature, causing higher velocity gradients near the hot wall and lower velocity gradients near the cold wall.

The mean temperature profiles are presented in Fig. 5b. For cases $\mathrm{SC}_{1}$ and SCCTP, the trans-critical transition occurs roughly between the middle of the channel and the hot wall, which is therefore a region of high $C_{p}$. The temperature profiles for $\mathrm{SC}_{1}$ and SCCTP are thus flat near the middle of the channel. On the other hand, for case $\mathrm{SC}_{2}$, the trans-critical transition occurs very close to the cold wall. So, the portions of the channel away from the cold wall are a region of low $C_{p}$, causing the temperature to change more rapidly with channel height away from the cold wall. The temperature in the bulk of the channel is therefore much higher than $T_{p c}$.

The mean density profiles are given in Fig. 5c. It can be observed that the density changes by a factor of around 2.5 from the hot wall to the cold wall. For the case $\mathrm{SC}_{1}$, the density changes steeply near both walls with a flatter profile near the middle of the channel. But, for the case $\mathrm{SC}_{2}$, the trans-critical transition very near to the cold wall results in the steep decline of density profile close to the cold wall and an approximately flat profile thereafter. It can be seen from Fig. $5 \mathrm{~b}$ that the temperature increases more rapidly near the cold wall for the case $\mathrm{SC}_{1}$ compared to SCCTP. At almost the same pressure, a faster rise in temperature corresponds to a faster decline in density. Thus, the density declines at a slower rate for $\mathrm{SCCTP}$ compared to $\mathrm{SC}_{1}$. This can explain the smaller rate of increase of density near the hot wall for SCCTP. Similarly, it is seen that the temperature near the hot wall decreases less rapidly for the case SCCTP.

By observing the root mean square (rms) density fluctuation profiles for cases $\mathrm{SC}_{1}$ and $\mathrm{SC}_{2}$ in Fig. 5d, it is evident that for $\mathrm{SC}_{1}$, the rms density fluctuation exhibits two peaks near the walls and a smaller peak near the middle of the channel. This behaviour is analogous to turbulent flows with ideal gas with wall temperature differences. This is however not the case for $\mathrm{SC}_{2}$ for which the rms density fluctuation has a very high value close to the cold wall and a much smaller peak near the middle of the channel. The very high density peak close to the cold wall can be attributed to inertial effects and the effects of trans-critical transition reinforcing each other very close to the cold wall. Due to the trans-critical transition near the cold wall, the temperature for most of the remaining channel height is higher than the pseudo-critical temperature. For temperatures far away from the pseudo-critical temperature, the change of density with change in temperature is minimal. Thus, the density profile is almost flat for locations away from the cold wall. This can be seen by observing the profiles of mean density in Fig. $5 \mathrm{c}$ for the case $\mathrm{SC}_{2}$. For the other cases, $\mathrm{SC}_{1}$ and SCCTP, the density has sharp gradients near both walls. Due to the absence of a significant gradient of mean density near the hot wall for $\mathrm{SC}_{2}$, the passive mixing across a mean gradient is minimal. This causes the peak of the density fluctuation near the hot wall to disappear for 

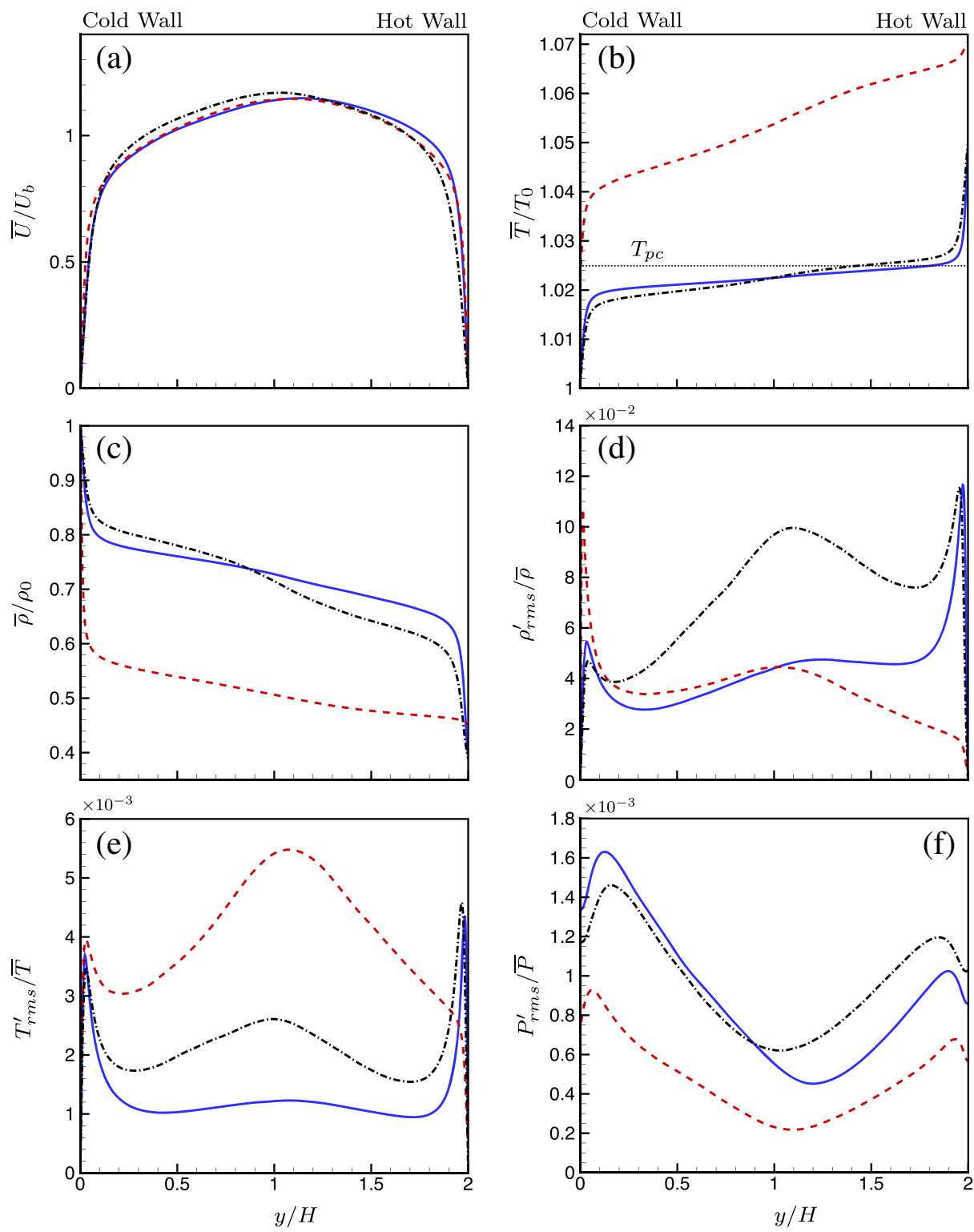

Fig. 5 Averaged quantities as a function of channel height for a mean velocity, $\mathbf{b}$ mean temperature, c mean density, $\mathbf{d}$ density rms, e temperature rms, and fpressure rms. ( - ) $\mathrm{SC}_{1} ;(---) \mathrm{SC}_{2} ;(---)$ SCCTP

$\mathrm{SC}_{2}$. Thus, a trans-critical transition very close to the wall causes this unusual behaviour in the density fluctuations. The rms temperature and the rms pressure fluctuations for the two cases are shown in Fig. 5e and f, respectively. The case $\mathrm{SC}_{2}$ has higher temperature fluctuations and lower pressure fluctuations near the center of the channel compared to the other two cases. 


\subsection{Near wall turbulence}

The streaks for the Favre averaged streamwise velocity, density, temperature and pressure near the hot and the cold walls of the channel are shown for $\mathrm{SC}_{2}$ in Fig. 6. The density, pressure and temperature streaks are normalized by their local mean values. The computational box is also scaled by the semi-local scales, $x^{*}$ and $z^{*}$. The semi-local coordinates
$\rho^{\prime} / \bar{\rho}$
$-0.003$
0.003
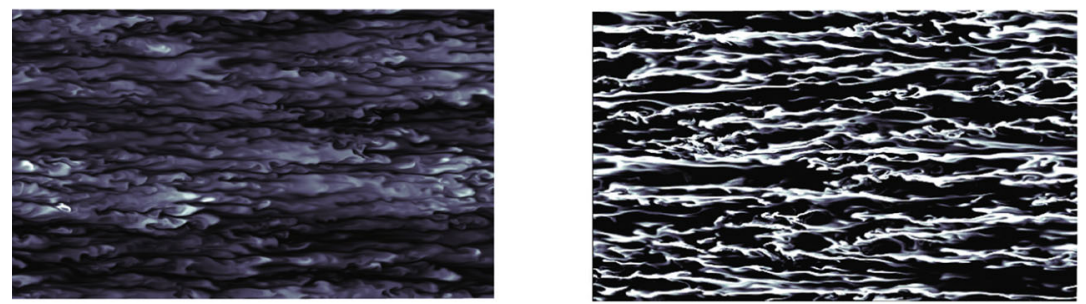

$T^{\prime} / \bar{T}$

$-0.005$

0.005
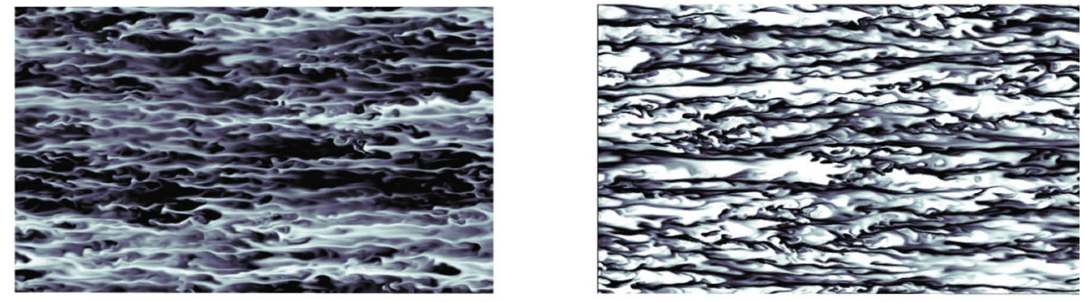

$p^{\prime} / \bar{p}$

$-0.1$

0.1
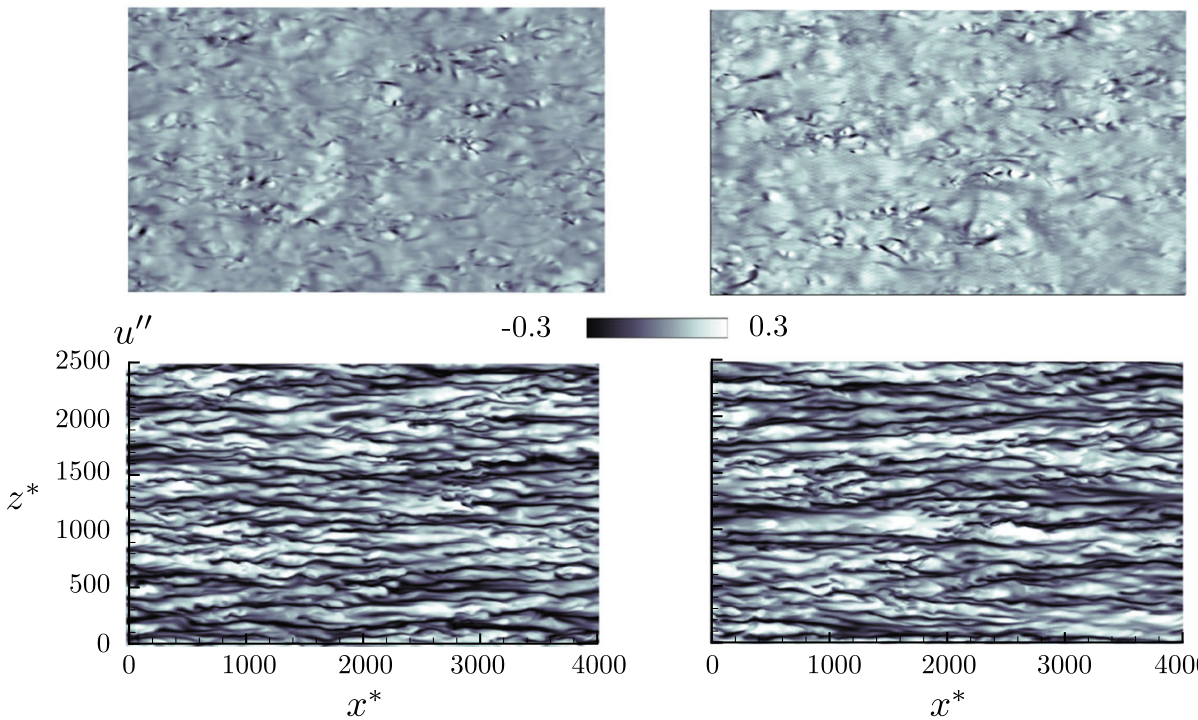

0.3

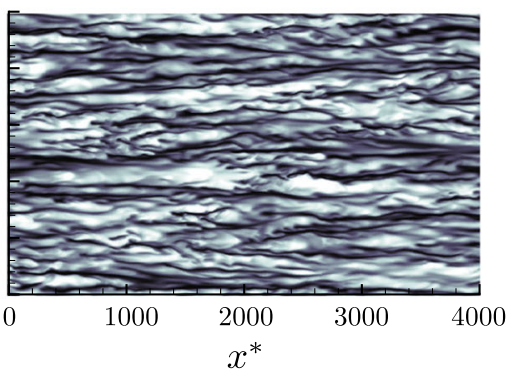

Fig. 6 Fluctuations for streamwise velocity $\left(u^{\prime \prime}\right)$, density $\left(\rho^{\prime} / \bar{\rho}\right)$, temperature $\left(T^{\prime} / \bar{T}\right)$, pressure $\left(p^{\prime} / \bar{p}\right)$ on the hot and cold sides of the channel for turbulent flows of supercritical $\mathrm{CO}_{2}\left(\mathrm{SC}_{2}\right)$. From top to bottom: density streaks, temperature streaks, pressure streaks, velocity streaks. Left column - hot wall $\left(y^{*}=14.72\right)$ and right column - cold wall $\left(y^{*}=16.98\right)$ 
are defined as $x^{*}=x \bar{\rho} u_{\tau}^{*} / \bar{\mu}$ (similarly for $y^{*}$ and $z^{*}$ ), with the semi-local friction velocity given as $u_{\tau}^{*}=\sqrt{\tau_{w} / \bar{\rho}}$. The streaks are calculated at the locations $y^{*}=14.72$ near the hot wall and $y^{*}=16.98$ near the cold wall based on the maxima for the root mean square velocity fluctuations near the respective walls. For this case, it is seen that there is no peak for the density fluctuation near the hot wall. This is due to the trans-critical transition very close to the cold wall. This behaviour is affirmed on analyzing the density fluctuation in Fig. 5d, when it is seen that near the hot wall there are almost no high density streaks, whereas near the cold wall high density streaks are more prevalent. The temperature streaks indicate the same behaviour to a lesser degree. Near the cold wall, there is a higher occurrence of high temperature fluctuations compared to that near the hot wall. The streaks also prove that, near the walls, the density is much more correlated with temperature compared to pressure, since no significant occurrences of high or low pressure fluctuations can be seen near the walls. The analysis of Favre averaged velocity fluctuations near the walls reveal that the high speed streaks are more enhanced near the cold wall compared to the hot wall. Also, the velocity streaks indicate more coherent structures near the cold wall.

\subsection{Mean velocity transformations}

For all of the above mentioned simulations, the walls are at different temperatures and the wall stresses at the cold and hot walls are different from one another. Therefore, the channel has been divided into hot and cold sides depending on the location of zero Reynolds shear stress. The locations of zero shear stress occur at $y / H=1.1488,1.0765,1.0172$ for the cases $\mathrm{SC}_{1}, \mathrm{SC}_{2}$ and $\mathrm{SCCTP}$, respectively. The laminar, turbulent and total shear stress on the hot and the cold sides of the channel, scaled by the wall stress on the respective sides, are shown in Fig. 7. The total shear stress shows a kink near the channel centre due to the loss of symmetry.

Based on [26], the changes in the semi-local Reynolds number, $R e_{\tau}^{*}$, defined as $R e_{\tau}^{*}=$ $\bar{\rho} u_{\tau}^{*} H / \bar{\mu}=\sqrt{\bar{\rho} / \rho_{w}} /\left(\bar{\mu} / \mu_{w}\right) R e_{\tau w}$, have a significant impact on inter-component energy transfer and hence on turbulence anisotropy in the flow field. The anisotropies are defined as $b_{i j}=\overline{u_{i}^{\prime} u_{j}^{\prime}} / \overline{u_{k}^{\prime} u_{k}^{\prime}}-1 / 3 \delta_{i j}$, where $\delta_{i j}$ is the Kronecker delta. When $d R e_{\tau}^{*} / d y<0$, it causes a reduction in momentum transfer and redistribution in turbulent kinetic energy from the streamwise direction to the other directions. This is evident in an increase in the streamwise anisotropy and a decrease in the spanwise anisotropy. The opposite behaviour is observed when $d R e_{\tau}^{*} / d y>0$. The semi-local Reynolds number $R e_{\tau}^{*}$ and the anisotropies
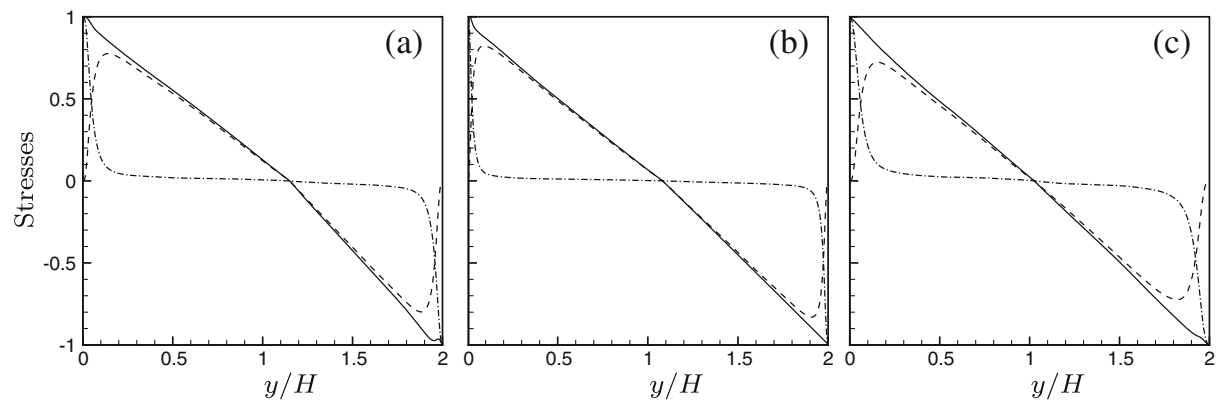

Fig. 7 Shear stresses as a function of channel height. $(---)-\bar{\rho} \overline{u^{\prime} v^{\prime}} / \tau_{w} ;(---)(\bar{\mu} \partial \bar{U} / \partial y) / \tau_{w}$; $(\longrightarrow)\left(-\bar{\rho} \overline{u^{\prime} v^{\prime}}+(\bar{\mu} \partial \bar{U} / \partial y)\right) / \tau_{w} \cdot \mathbf{a} \mathrm{SC}_{1} ; \mathbf{b ~ S C} 2 ; \mathbf{c ~ S C C T P}$ 
$b_{u u}, b_{w w}$ and $b_{v v}$ in the streamwise, spanwise and wall-normal directions, respectively, are show for the three simulations in Fig. 8. It is seen from Fig. 8a and g, that for $\mathrm{SC}_{1}$, the streamwise anisotropy on the cold side is less than that on the hot side of the channel. This can be explained by the fact that $d R e_{\tau}^{*} / d y>0$ on the cold side of the channel and $d R e_{\tau}^{*} / d y<0$ on the hot side of the channel. So, there is a reduction in redistribution of turbulent kinetic energy on the hot side and an increase on the cold side of the channel. The opposite is observed for the streamwise anisotropy in SCCTP as $R e_{\tau}^{*}$ is decreasing on the cold side of the channel and increasing on the hot side of the channel. This is demonstrated in Fig. $8 \mathrm{c}$ and i. From Fig. $8 \mathrm{~b}$ and h, it is observed that, for $\mathrm{SC}_{2}$, the anisotropies are nearly equal on the two sides of the channel due to the flatter $R e_{\tau}^{*}$ profiles as a result of which $d R e_{\tau}^{*} / d y$ is almost equal to zero for most of the channel height. For all the cases, it is seen that an increase in streamwise anisotropy is accompanied with a corresponding decrease in the spanwise anisotropy, while the anisotropy in the wall-normal direction in the hot and cold sides of the channel seems to be relatively unaffected, whether $R e_{\tau}^{*}$ is increasing or decreasing. The van Driest transformation has been previously used to scale the streamwise velocity $\left(\bar{u}^{V D}\right)$ in compressible flows. This provides a reasonable collapse of the velocity profiles with the log law of the wall. A more recent approach is the extended van Driest transformation developed by [27] and [28]. The extended van Driest transformed velocity $\left(\overline{u^{*}}\right)$ is plotted as a function of $y^{*}$ for the three simulations in Fig. 8d, e and f. This is seen to produce a fairly good collapse of the velocity profiles with the log law for the wall.
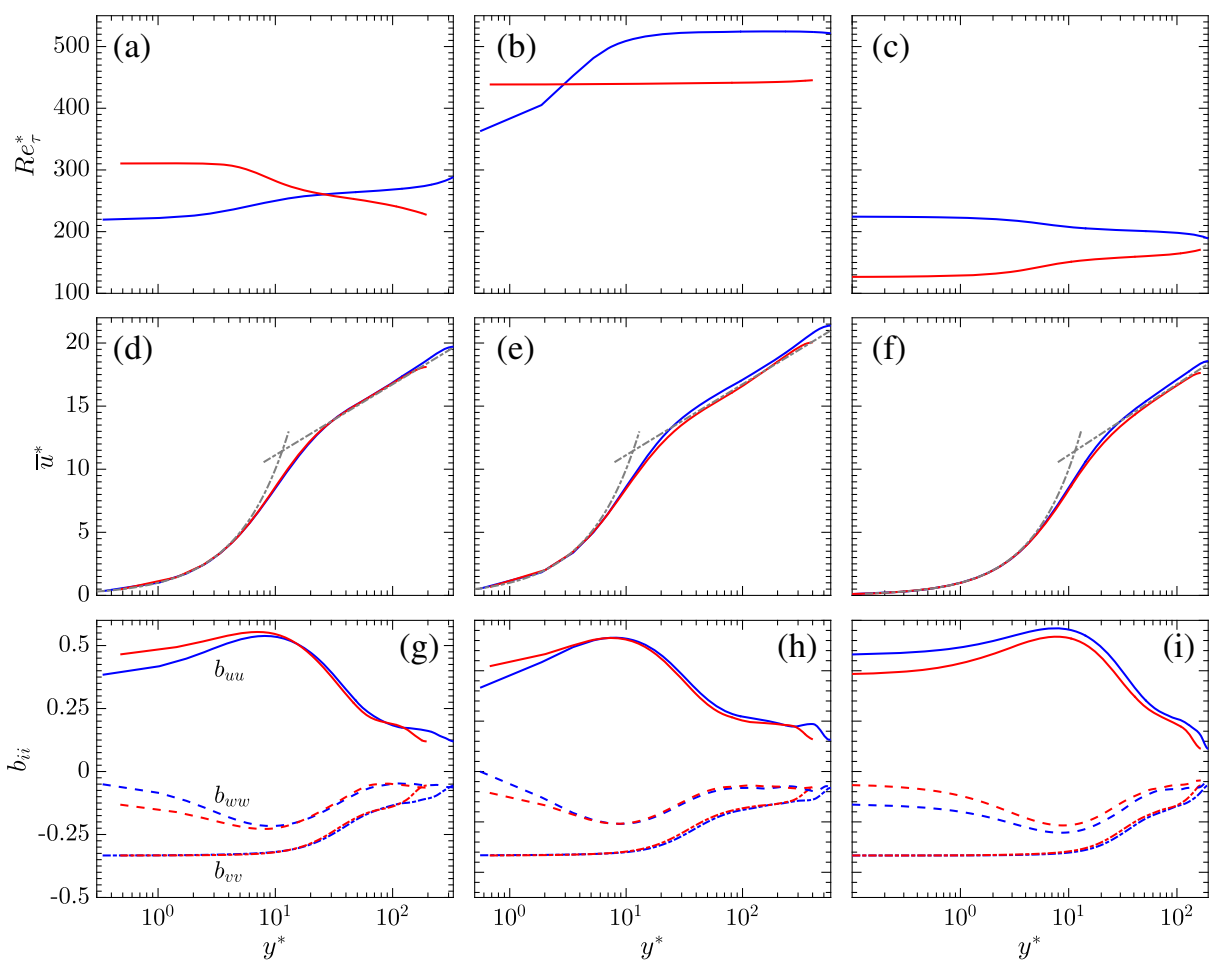

Fig. $8 R e_{\tau}^{*}$ (top row), extended van Driest transformed velocity (middle row), and turbulence anisotropies (bottom row) as a function of $y^{*}$; (left column) $\mathrm{SC}_{1}$, (middle column) $\mathrm{SC}_{2}$, (right column) SCCTP; ( cold side, $(\longrightarrow)$ hot side 


\subsection{Semi locally scaled turbulent statistics and budgets}

The velocity fluctuations and the quantities involved in evaluating the budgets for the turbulent kinetic energy $\left(k=1 / 2 \bar{\rho} \widehat{u_{i}^{\prime \prime} u_{i}^{\prime \prime}}\right)$ are scaled by the semi-local variables and plotted as a function of $y^{*}$. The budget equation used is the same as that given by [29] and [30], and is given as follows,

$$
P_{k}+D_{k}+\epsilon_{k}+C_{k}=0,
$$

with the turbulent production $P_{k}$, diffusion $D_{k}$, dissipation $\epsilon_{k}$ and compressibility terms $C_{k}$. These are expressed as

$$
\begin{gathered}
P_{k}=-\widetilde{\rho} \widetilde{u^{\prime \prime} v^{\prime \prime}} \frac{\partial \tilde{u}}{\partial y} ; D_{k}=\frac{\partial\left[\overline{\Gamma_{i 2}^{\prime} u_{i}^{\prime}}-\bar{\rho} \widetilde{v^{\prime \prime} u_{i}^{\prime \prime} u_{i}^{\prime \prime}}-\overline{p^{\prime} v^{\prime}}\right]}{\partial y} \\
\epsilon_{k}=-\Gamma_{i j}^{\prime} \frac{\partial u_{i}^{\prime}}{\partial x_{j}} ; C_{k}=-C_{k 1}+C_{k 2}+C_{k 3} \\
C_{k 1}=\overline{v^{\prime \prime}} \frac{\partial \bar{p}}{\partial y} ; C_{k 2}=\overline{u_{i}^{\prime \prime} \frac{\partial \Gamma_{i 2}}{\partial y}} ; C_{k 3}=\overline{p^{\prime} \frac{\partial u_{k}^{\prime}}{\partial x_{k}}} .
\end{gathered}
$$

The velocity fluctuations and the turbulent kinetic energy budgets scaled by the semi-local variables are shown for the three cases in Fig. 9. The velocity fluctuations and the turbulent kinetic energy budgets, if scaled by bulk variables do not collapse on top of each other. However, the values on the hot and cold sides of the channel are found to collapse when scaled by semi-local variables. The validity of the semi-local scaling also reaffirms the relevance of the Morkovin hypothesis for all of the simulations performed. This signifies that the changes in the turbulence structures are due to changes in mean density and mean viscosity gradients and fluctuations of these quantities have a limited impact on the turbulence in the flow.
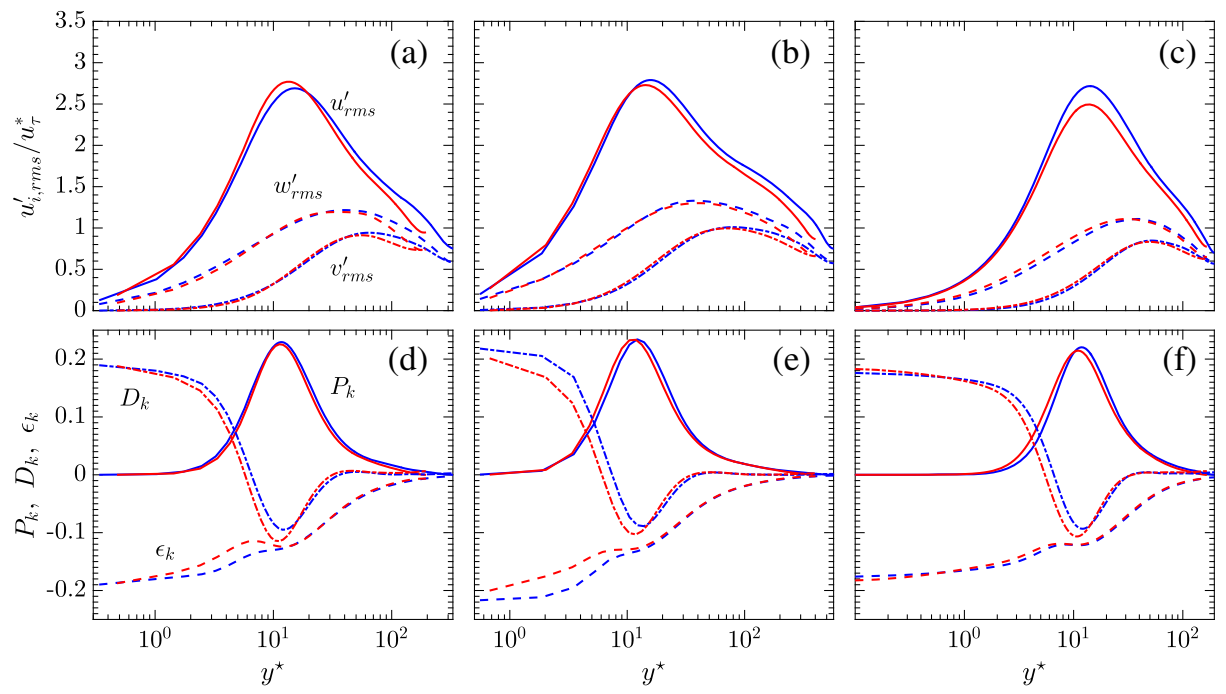

Fig. 9 Semi-locally scaled velocity fluctuations (top row) and turbulent kinetic energy budgets (bottom row) as a function of $y^{*}$. (left column) $\mathrm{SC}_{1}$, (middle column) $\mathrm{SC}_{2}$, (right column) SCCTP; ( $\longrightarrow$ ) cold side, $(-)$ hot side 


\subsection{Inertial and viscous effects}

As the flow is compressible in nature with variable viscosity, the turbulence in the flow is influenced both by the variable inertial and viscous effects on the hot and cold side of the channel. The magnitudes of the inertial and viscous forces on the hot and cold sides of the channel were quantified by [25]. These are given as follows,

$$
F_{I}^{h} \propto \rho_{b}^{h} U_{b}^{h}\left(h^{h}\right)^{2} ; F_{I}^{c} \propto \rho_{b}^{c} U_{b}^{c}\left(h^{c}\right)^{2}
$$

where $\left(F_{I}^{h} F_{I}^{c}\right),\left(\rho_{b}^{h} \rho_{b}^{c}\right),\left(U_{b}^{h} U_{b}^{c}\right)$ and $\left(h^{h} h^{c}\right)$ refer to the inertial force, the bulk density, the bulk velocity and the channel height on the hot and cold sides of the channel, respectively.

$$
F_{V}^{h} \propto \mu_{b}^{h} U_{b}^{h} h^{h} ; F_{V}^{c} \propto \mu_{b}^{c} U_{b}^{c} h^{c}
$$

where $\left(F_{V}^{h} F_{V}^{c}\right)$ and $\left(\mu_{b}^{h} \mu_{b}^{c}\right)$ refer to the viscous forces and the bulk viscosity on the hot and cold sides of the channel, respectively. The ratio of inertial and viscous forces on the hot and cold sides of the channel are given in Table 4.

This shows that in all the cases, there is an increase in the viscous forces from the hot to the cold side of the channel, but there is an even bigger increase in inertial forces. This is due to the fact that the inertial force is proportional to the square of the channel height and the channel height on the cold side is greater than the channel height on the hot side for each of the cases simulated. Also, for the constant viscosity case, the ratio of the viscous forces on the two sides of the channel is found to be close to unity. This is not the case with the variable viscosity cases.

\subsection{Compressibility effects}

Previous authors, such as [30, 31] have divided the turbulent kinetic energy dissipation into the sum of the solenoidal, dilatational and inhomogeneous parts. The solenoidal dissipation $\left(\epsilon_{s}=-\bar{\mu} \overline{\omega_{i j}^{\prime} \omega_{i j}^{\prime}}\right)$ represents the pseudo-dissipation for the corresponding incompressible flow, where $\omega$ is used to refer to the vorticity in the flow field. The inhomogeneous component of the dissipation is found to be neglible. The dilatational dissipation is the part of the total dissipation attributed to compressibility effects. So, the total dissipation can be divided into the sum of the incompressible and compressible parts, namely, the solenoidal and the dilatational dissipation. The compressibility effects are thereby evaluated by comparing the solenoidal dissipation to the total dissipation for turbulent kinetic energy $\left(\epsilon_{k}\right)$. Both of these quantities on the hot and cold sides of the channel are scaled by the semi-local variables and are plotted in Fig. 10. If compressibility effects are minimal, the solenoidal dissipation and the total dissipation should almost collapse on top of each other. The biggest difference between the solenoidal and the total dissipation is observed in the case $\mathrm{SC}_{2}$ very near to the cold wall. The other cases $\mathrm{SC}_{1}$ and SCCTP with trans-critical transition near the middle of the channel do not exhibit significant levels of compressibility close to the walls as the solenoidal and total dissipations almost collapse on top of each other. So, for turbulent flows of supercritical $\mathrm{CO}_{2}$, it is possible to experience significant levels of compressibility

Table 4 Ratio of the inertial and viscous forces on the hot and cold sides of the channel

\begin{tabular}{lll}
\hline Case & $F_{I}^{c} / F_{I}^{h}$ & $F_{V}^{c} / F_{V}^{h}$ \\
\hline $\mathrm{SC}_{1}$ & 2.03 & 1.60 \\
$\mathrm{SC}_{2}$ & 1.57 & 1.23 \\
$\mathrm{SCCTP}$ & 1.39 & 1.06 \\
\hline
\end{tabular}



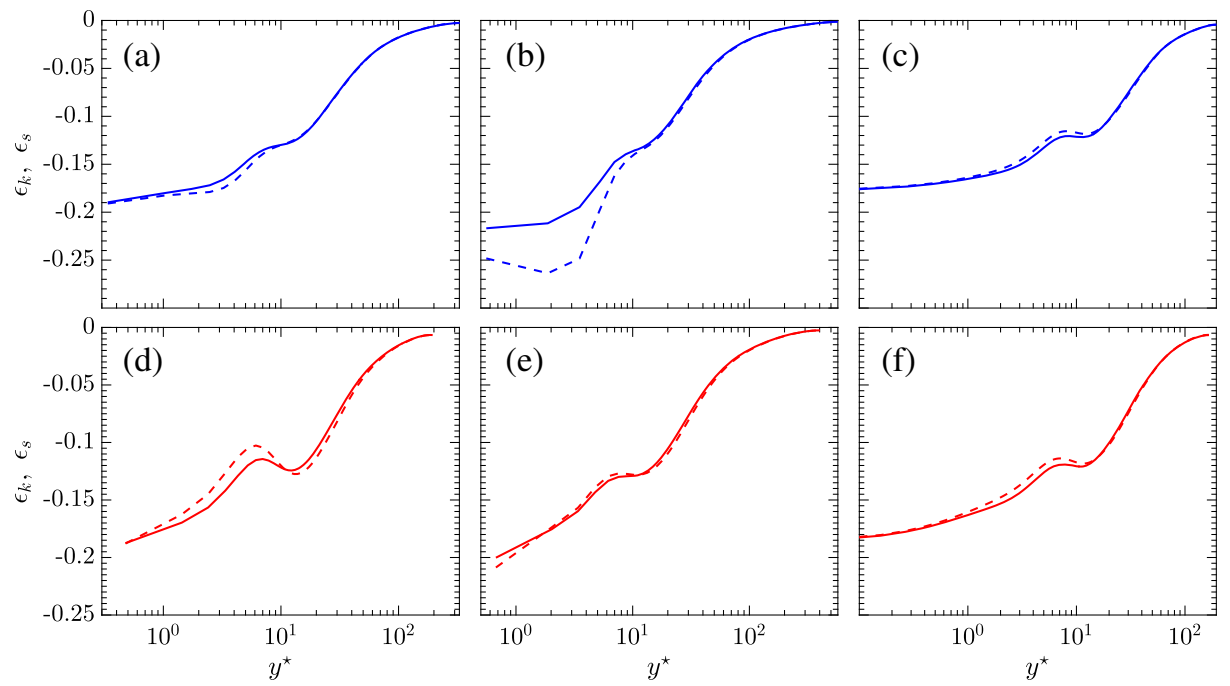

Fig. 10 Total $\epsilon_{k}(-)$ and solenoidal $\epsilon_{s}(---)$ dissipation as a function of $y^{*}$; (left column) $\mathrm{SC}_{1}$, (middle column) $\mathrm{SC}_{2}$, (right column) SCCTP; (- $(-)$ cold side, $\left(-\frac{}{-}\right)$ hot side

caused by trans-critical transition near to the cold wall. But, overall, the compressibility effects experienced are still small as they are significant only in the case of trans-critical transition very close to the wall and are confined to a limited region close to the wall. It is expected that these effects will be more pronounced for higher Mach number cases or for pressures closer to the critical pressure.

\section{Conclusion}

Fully compressible direct numerical simulations are performed for turbulent flows of supercritical $\mathrm{CO}_{2}$ at low Mach numbers close to the vapour-liquid critical point involving trans-critical transition. The main conclusions from our research are as follows.

The location of trans-critical transition has an influence on the mean and turbulent statistics. Comparison of the mean temperature and the mean density profile for the cases $\mathrm{SC}_{1}$ and $\mathrm{SC}_{2}$, reveal significant differences. These are due to the changing nature of the density and the isobaric heat capacity for $\mathrm{CO}_{2}$. When the pseudo-critical temperature is located very close to the cold wall, as for the case $\mathrm{SC}_{2}$, it is seen to alter the traditional nature of turbulence. This is seen in the unusual nature of the density fluctuations, which is not observed in turbulent ideal gas flows with a wall temperature difference. The analysis of the streaks for density and temperature for $\mathrm{SC}_{2}$ also indicate that the cold wall region is characterized by much higher occurrences of high temperature and high density fluctuations than the region near the hot wall. The streamwise velocity streaks for $\mathrm{SC}_{2}$ near the hot and cold walls signify that high speed streaks are much more prevalent near the cold wall and also, the structures near the cold wall have a higher degree of coherence. A comparison of the semi-locally scaled solenoidal and total dissipation indicates that trans-critical transition very close to the wall can produce compressibility effects, even at low Mach numbers. This is observed from the significant differences between solenoidal and total dissipation close to the cold wall for $\mathrm{SC}_{2}$. But, the compressibility effects in the overall perspective are quite small as 
these are only confined to a small part of the channel very close to the cold wall and also, for all the other simulations, they almost collapse on top of each other. The compressibility effects should become even more accentuated for higher Mach number cases.

The change in the nature of the $R e_{\tau}^{*}$ profiles is has a significant impact on the redistribution of turbulent kinetic energy. This is evident in the fact that, on comparing the streamwise anisotropies on the hot and cold sides of the channel, the anisotropy is seen to increase when $d R e_{\tau}^{*} / d y<0$ due to which there is a reduction in the redistribution of turbulent kinetic energy form the streamwise to the other directions. The opposite behaviour is observed when $d R e_{\tau}^{*} / d y>0$. Thus, we can conclude that liquid-like behaviour which is characterized by an increasing $R e_{\tau}^{*}$ causes a decrease in the streamwise anisotropy and an increase in the spanwise anisotropy due to the increase in the turbulent kinetic energy redistribution from streamwise to the other directions, and the opposite is seen with gas-like behaviour.

The semi-locally scaled velocity fluctuations and turbulent kinetic energy budgets on the hot and cold sides of the channel are found to collapse almost on top of each other. This result is seen to reinforce Morkovin's hypothesis as it establishes that the changes in the turbulence structure are only caused by the change in the mean density gradients in the flow and the influence of density fluctuations on the changes in turbulence structures is insignificant.

Acknowledgments We acknowledge that the results of this research have been accomplished by using the computational resources of SURFsara in The Netherlands. Also, we would like to acknowledge the contribution of FOM (NWO) in the Netherlands for funding this research.

\section{Compliance with Ethical Standards}

Conflict of interests The authors declare that they have no conflict of interest.

Open Access This article is distributed under the terms of the Creative Commons Attribution 4.0 International License (http://creativecommons.org/licenses/by/4.0/), which permits unrestricted use, distribution, and reproduction in any medium, provided you give appropriate credit to the original author(s) and the source, provide a link to the Creative Commons license, and indicate if changes were made.

\section{Appendix}

\section{Appendix A: Derivation of departure functions}

The non-dimensional van der Waals $(\mathrm{VdW})$ equation of state is given as:

$$
P=\frac{R T}{\vartheta-b}-\frac{a}{\vartheta^{2}},
$$

where $P$ is the pressure, $T$ is the temperature, $\vartheta$ is the specific volume. The nondimensionalization is performed as follows:

$$
R=\frac{R^{\star} T_{0}^{\star}}{U_{0}^{\star 2}} ; a=\frac{a^{\star} \rho_{0}^{\star}}{U_{0}^{\star 2}} ; b=b^{\star} \rho_{0}^{\star} .
$$

The dimensional constants $a^{\star}$ and $b^{\star}$ are determined by the critical properties of the real gas. These constants are given as:

$$
a^{\star}=3 P_{c}^{\star}\left(V_{c}^{\star 2}\right) ; b^{\star}=V_{c}^{\star} / 3,
$$


where $P_{c}^{\star}$ is the critical pressure and $V_{c}^{\star}$ is the critical volume of the gas. The residual internal energy $\left(U^{R}\right)$ is given as:

$$
U^{R}=U-U^{i g}=\int_{\infty}^{\vartheta}\left(T\left(\frac{\partial S}{\partial \vartheta}\right)_{T}-P\right) d \vartheta
$$

Using Maxwell's relations, we get: $(\partial S / \partial \vartheta)_{T}=(\partial P / \partial T)_{\vartheta}$. Substituting in the integral, $U^{R}=\int_{\infty}^{\vartheta}\left(T(\partial P / \partial T)_{\vartheta}-P\right) d \vartheta$. Furthermore, from the van der Waals equation: $(\partial P / \partial T)_{\vartheta}=R /(\vartheta-b)$. The expression for the residual internal energy is thereby given as: $U^{R}=\int_{\infty}^{\vartheta}\left(a / \vartheta^{2}\right) d \vartheta$. This has also been given in the works published by previous authors, such as [32,33]. Thus, we get:

$$
U-C_{\vartheta} T=-\frac{a}{\vartheta} ; U=C_{\vartheta} T-a \rho,
$$

where $C_{\vartheta}$ is the heat capacity of the gas at constant volume.

The non-dimensional Redlich Kwong (RK) equation of state is given as:

$$
P=\frac{R T}{\vartheta-b}-\frac{a \alpha}{(\vartheta)(\vartheta+b)}
$$

The dimensional constants $a^{\star}$ and $b^{\star}$ are given as: $a^{\star}=\left(0.42748 R^{\star 2} T_{c}^{\star 2.5}\right) /\left(P_{c}\right), b^{\star}=$ $\left(0.08662 R^{\star} T_{c}^{\star}\right) /\left(P_{c}^{\star}\right)$. The parameter $\alpha$ is given as: $\alpha=1 / \sqrt{T^{\star}}$.

For this equation of state, following the same procedure as mentioned above, we get,

$$
T\left(\frac{\partial P}{\partial T}\right)_{\vartheta}-P=\frac{a\left(\alpha-T \frac{d \alpha}{d T}\right)}{(\vartheta)(\vartheta+b)} .
$$

So, the residual internal energy is given as:

$$
U^{R}=\int_{\infty}^{\vartheta}\left(T\left(\frac{\partial P}{\partial T}\right)_{\vartheta}-P\right)=\frac{a\left(\alpha-T \frac{d \alpha}{d T}\right)}{b} \log \frac{\vartheta}{\vartheta+b}
$$

Substituting the value of $\alpha$

$$
U=C_{\vartheta} T+\frac{3}{2} \frac{a}{b \sqrt{T}} \log \frac{1}{1+b \rho} .
$$

\section{Appendix B: Validation}

The compressible Navier-Stokes code has been validated with the data published by previous authors for both low Mach number [22] and high Mach number [23] cases. This is shown in the Figs. 11 and 12 with the help of both mean and turbulent statistics. The low Mach number and high Mach number cases used for validating the code are henceforth referred to as KMM and CKM, respectively. The corresponding simulations carried out with our present code are called Ma0.2 and Ma1.5. The details of these simulations are given in Table 5. The plots in Figs. 11 and 12 show a fairly good agreement for the mean flow profiles, the turbulent velocity fluctuations and the Reynolds shear stress with the data published by the previous authors. 

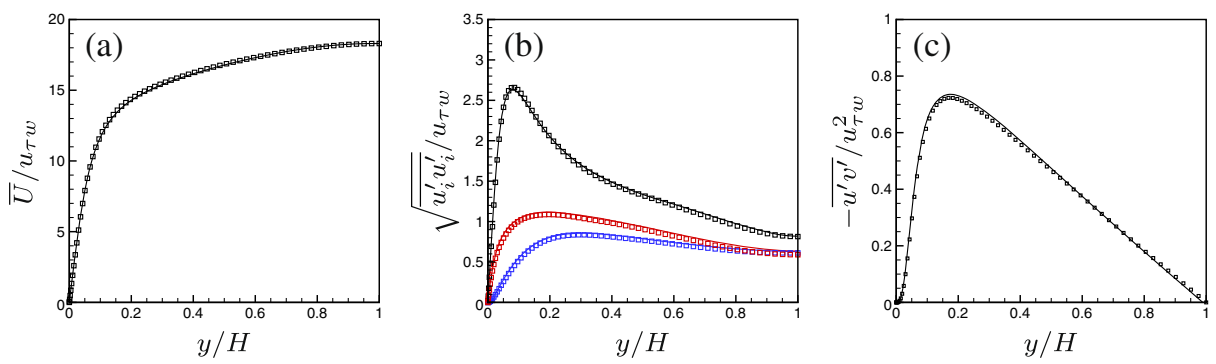

Fig. 11 Validation of the compressible DNS code [22]; Lines: present; Symbols: KMM
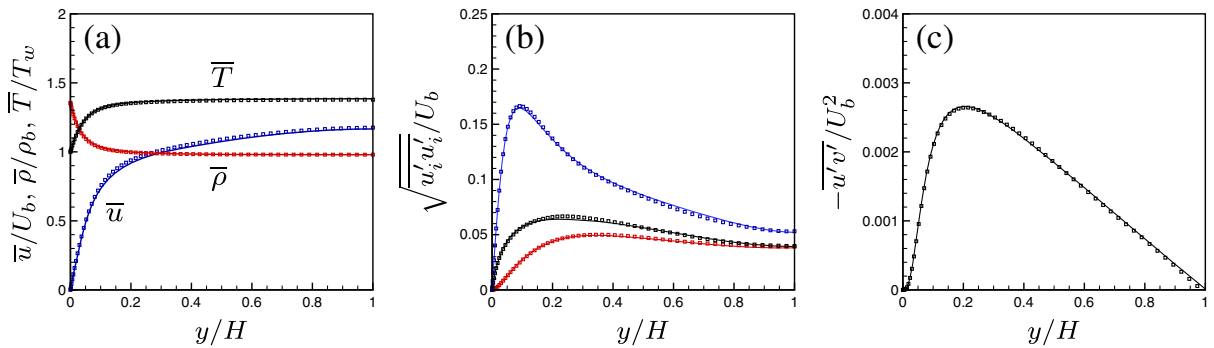

Fig. 12 Validation of the compressible DNS code [23]; Lines: present; Symbols: CKM

Table 5 Validation of the code for low and high Mach number cases using ideal gas equation of state; $M a$ : Mach number; $R e$ : bulk Reynolds number; $R e_{\tau}$ : friction Reynolds number at the wall; $\operatorname{Pr}$ : Prandtl number; $N_{x}, N_{y}, N_{z}$ : number of grid points; $L_{x}, L_{y}, L_{z}$ : lengths of channel

\begin{tabular}{lllllll}
\hline Case & $M a$ & $R e$ & $R e_{\tau}$ & $P r$ & $N_{x} \times N_{y} \times N_{z}$ & $L_{x} \times L_{y} \times L_{z}$ \\
\hline KMM & 0.0 & 2800 & 178 & 0.7 & $192 \times 129 \times 160$ & $4 \pi h \times 2 h \times \frac{4}{3} \pi h$ \\
CKM & 1.5 & 3000 & 222 & 0.7 & $144 \times 119 \times 80$ & $4 \pi h \times 2 h \times \frac{4}{3} \pi h$ \\
Ma0.2 & 0.2 & 2800 & 180 & 0.7 & $120 \times 168 \times 120$ & $4 \pi h \times 2 h \times 2 \pi h$ \\
Ma1.5 & 1.5 & 3000 & 209 & 0.7 & $120 \times 168 \times 120$ & $4 \pi h \times 2 h \times 2 \pi h$ \\
\hline
\end{tabular}




\section{References}

1. Lemmon, E.W., Huber, M.L., McLinden, M.O.: NIST Standard ReferenceDatabase 23 Reference Fluid Thermodynamic and Transport Properties - REFPROP 9.0. (2010)

2. Zonta, F.: Nusselt number and friction factor in thermally stratified turbulent channel flow under nonoberbeck-boussinesq conditions. Int. J. Heat Fluid Flow 44, 489-494 (2013)

3. Zonta, F., Soldati, A.: Effect of temperature dependent fluid properties on heat transfer in turbulent mixed convection. J. Heat Transfer 136(2), 022501 (2014)

4. Bae, J.H., Yoo, J.Y., McEligot, D.M.: Direct numerical simulation of heated $\mathrm{CO}_{2}$ flows at supercritical pressure in a vertical annulus at $\mathrm{Re}=8900$. Phys. Fluids 20, 055108 (2008)

5. Schmitt, T., Selle, L., Ruiz, A., Cuenot, B.: Large-eddy simulation of supercritical-pressure round jets. Amer. Inst. Aeronaut. Astronaut. J. 48(9), 2133-2144 (2010)

6. He, S., Kim, W.S., Bae, J.H.: Assessment of performance of turbulence models in predicting supercritical pressure heat transfer in a vertical tube. Int. J. Heat Mass Transfer 51(19), 4659-4675 (2008)

7. Yoo, J.Y.: The turbulent flows of supercritical fluids with heat transfer. Ann. Rev. Fluid Mech. 45, 495525 (2013)

8. Kawai, S., Terashima, H., Negishi, H.: A robust and accurate numerical method for transcritical turbulent flows at supercritical pressure with an arbitrary equation of state. J. Comput. Phys. 300, 116-135 (2015)

9. Nemati, H., Patel, A., Boersma, B.J., Pecnik, R.: Mean statistics of a heated turbulent pipe flow at supercritical pressure. Int. J. Heat Mass Transfer 83, 741-752 (2015)

10. Chu, X., Laurien, E.: Flow stratification of supercritical co 2 in a heated horizontal pipe. J. Supercrit. Fluids 116, 172-189 (2016)

11. Müller, H., Niedermeier, C.A., Matheis, J., Pfitzner, M., Hickel, S.: Large-eddy simulation of nitrogen injection at trans-and supercritical conditions. Phys. Fluids 28(1), 015102 (2016)

12. Alferez, N., Touber, E.: One-dimensional refraction properties of compression shocks in non-ideal gases. J. Fluid Mech. 814, 185-221 (2017)

13. Peeters, J.W.R., Pecnik, R., Rohde, M., van der Hagen, T.H.J.J., Boersma, B.J.: Characteristics of turbulent heat transfer in an annulus at supercritical pressure. Phys. Rev. Fluids 2(2), 024602 (2017)

14. Span, R., Wagner, W.: Equations of state for technical applications. I. Simultaneously optimized functional forms for nonpolar and polar fluids. Int. J. Thermophys. 24, 1-39 (2003). ISSN 0195-928X

15. Kunz, O., Wagner, W.: The GERG-2008 wide-range equation of state for natural gases and other mixtures: an expansion of GERG-2004. J. Chem. Eng. Data 57(11), 3032-3091 (2012)

16. Swesty, F.D.: Thermodynamically consistent interpolation for equation of state tables. J. Comput. Phys. 127(1), 118-127 (1996). ISSN 0021-9991

17. Fenghour, A., Wakeham, W.A., Vesovic, V.: The viscosity of carbon dioxide. J. Phys. Chem. Ref. Data 27(1), 31-44 (1998)

18. Vesovic, V., Wakeham, W.A., Olchowy, G.A., Sengers, J.V., Watson, J.T.R., Millat, J.: The transport properties of carbon dioxide. J. Phys. Chem. Ref. Data 19(3), 763-808 (1990)

19. Morinishi, Y.: Skew-symmetric form of convective terms and fully conservative finite difference schemes for variable density low-mach number flows. J. Comput. Phys. 229(2), 276 - 300 (2010). ISSN 0021 9991

20. Lele, S.K.: Compact finite difference schemes with spectral-like resolution. J. Comput. Phys. 103(1), 16-42 (1992)

21. Gottlieb, S., Shu, C.W.: Total variation diminishing runge-kutta schemes. Math. Comput. Amer. Math. Soc. 67(221), 73-85 (1998)

22. Kim, J., Moin, P., Moser, R.: Turbulence statistics in fully developed channel flow at low reynolds number. J. Fluid Mech. 177, 133-166 (1987)

23. Coleman, G.N., Kim, J., Moser, R.D.: A numerical study of turbulent supersonic isothermal-wall channel flow. J. Fluid Mech. 305, 159-183 (1995)

24. Lee, J., Yoon Jung, S., Jin Sung, H., Zaki, T.A.: Effect of wall heating on turbulent boundary layers with temperature-dependent viscosity. J. Fluid Mech. 726, 196-225 (2013)

25. Zonta, F., Marchioli, C., Soldati, A.: Modulation of turbulence in forced convection by temperaturedependent viscosity. J. Fluid Mech. 697, 150-174 (2012)

26. Patel, A., Peeters, J.W.R., Boersma, B.J., Pecnik, R.: Semi-local scaling and turbulence modulation in variable property turbulent channel flows. Phys. Fluids (1994-present) 27(9), 095101 (2015)

27. Trettel, A., Larsson, J.: Mean velocity scaling for compressible wall turbulence with heat transfer. Phys. Fluids 28(2), 026102 (2016)

28. Patel, A., Boersma, B.J., Pecnik, R.: The influence of near-wall density and viscosity gradients on turbulence in channel flows. J. Fluid Mech. 809, 793-820 (2016) 
29. Morinishi, Y., Tamano, S., Nakabayashi, K.: Direct numerical simulation of compressible turbulent channel flow between adiabatic and isothermal walls. J. Fluid Mech. 502, 273-308 (2004)

30. Huang, P.G., Coleman, G.N., Bradshaw, P.: Compressible turbulent channel flows: DNS results and modelling. J. Fluid Mech. 305(1), 185-218 (1995)

31. Kreuzinger, J., Friedrich, R., Gatski, T.B.: Compressibility effects in the solenoidal dissipation rate equation A priori assessment and modeling. Int. J. Heat Fluid Flow 27(4), 696-706 (2006)

32. Elliott, J.R., Lira, C.T.: Introductory chemical engineering thermodynamics. Prentice Hall PTR, Upper Saddle River (1999)

33. Poling, B.E., Prausnitz, J.M., O'connell, J.P., et al.: The properties of gases and liquids, vol. 5. Mcgrawhill, New York (2001) 